\title{
Editorial
}

\section{The importance of epidermal stem cells in keratinocyte-mediated gene therapy}

\section{Cultivation of transplantable sheets of human epidermis} Epidermis, the outermost layer of skin, consists of a stratified squamous epithelium composed of a main cell type, the keratinocyte.

Keratinocytes form distinct cellular layers. The basal layer is composed of keratinocytes with a proliferative capacity and contains stem cells, namely cells that upon division replace their own number and also give rise to cells that differentiate further into one or more specialized cell types. Basal cells that become committed to terminal differentiation lose their proliferative capacity, migrate upwards and initiate the process of programmed cell death, leading to the sequential formation of the stratum spinosum, the stratum granulosum and the stratum corneum, the latter being composed of numerous layers of tightly packed and strongly flattened cells crucial for the epidermal barrier function.

Primary human keratinocytes isolated in culture form colonies, each colony being the progeny of a single clonogenic basal keratinocyte. Keratinocyte colonies will eventually fuse and differentiate giving rise to a stratified squamous epithelium, routinely used to make autologous grafts for patients suffering from large skin defects. ${ }^{1}$ The engrafted stem cells ensure the persistence of the regenerated epidermis during the patient's lifetime.

These characteristics fulfil two of the most important requirements for a cell to be the target of ex vivo gene therapy: (1) the possibility of easy cultivation; and (2) the availability of surgical protocols for grafting autologous cultured cells back on to patients.

\section{Rationale for the use of human keratinocytes in gene therapy}

Basal epidermal keratinocytes rest on a basal lamina composed of specific extracellular matrix proteins (Ref. 2 and references herein). ${ }^{2}$ The firm adhesion of basal keratinocytes, hence of the whole epidermis, to the basal lamina is mediated by hemidesmosomes. These structures link the epithelial intermediate filament network to the dermal anchoring fibrils, which are mainly composed of type VII collagen and extend from the basement membrane to anchoring plaques in the papillary dermis. ${ }^{2}$ These processes are also mediated by integrin receptors, a class of trans-membrane glycoproteins composed of $\alpha$ and $\beta$ chains. In particular, the $\alpha 6 \beta 4$ integrin, which is the only integrin in hemidesmosomes of stratified squamous and transitional epithelia, is the receptor for the basal lamina component laminin 5 .

Epidermolysis bullosa (EB) is an heritable devastating blistering disorder of the skin divided into three major categories. $^{2}$ In the simplex forms of EB the tissue separation occurs within the basal keratinocytes and is mainly due to mutations in keratin genes forming the intermediate filament network. In the junctional forms of EB, the tissue cleavage occurs within the dermal-epidermal junction and is due to mutations in genes encoding the $\alpha 3$, $\beta 3$ and $\gamma 2$ chains of laminin 5, the two subunits of the $\alpha 6 \beta 4$ integrin and the hemidesmosome-associated 180$\mathrm{kDa}$ bullous pemphigoid antigen. In the dystrophic forms of EB, the tissue cleavage occurs at the level of the anchoring fibrils and is due to mutations in the type VII collagen gene. Some forms of junctional EB, such as the Herlitz type, are usually lethal, while non-Herlitz junctional EB and dystrophic EB lead to disfiguring scars and severely impair the quality of life of patients.

Since basal keratinocytes are responsible for the synthesis of most of the above proteins, it is obvious that any attempt at gene therapy for the treatment of these genetic skin diseases requires the genetic modification of basal keratinocytes.

The importance of epidermal stem cells in gene therapy The epidermis is completely renewed approximately every month.

To accomplish this, the epidermis relies on stem and transient amplifying cells. Independent of its multipotent (as for the haematopoietic stem cell) or unipotent (as for the epidermal stem cell) nature, the basic and indispensable characteristic of a stem cell is its capacity for selfrenewal potential extending for at least one life-span of the organism. In skin, transient amplifying cells, which arise from stem cells and eventually generate terminally differentiated cells and have a high proliferative rate for only a limited period of time, represent the largest group of dividing cells and deal with the emergency of wound healing.

It is therefore self-evident that any attempt at using keratinocytes for gene therapy requires: (1) ex vivo cultivation of epidermal stem cells; and (2) stable integration of the exogenous gene into the epidermal stem cell genome.

The long-term follow-up of patients with burns treated with autologous cultivated epidermal sheets is the best demonstration that, under the appropriate culture conditions, stem cells can be maintained in culture and grafted back to patients. However, a large amount of data generated during the last 10 years using retrovirus- or 
adenovirus-mediated gene transfer has failed to demonstrate permanent transgene expression in normal human keratinocytes either in vitro or in vivo (Refs 3, 4 and references herein). Since grafts derived from a transduced human epidermal immortal keratinocyte cell line are able to sustain secretion of the transgene product for a relatively long period in vitro and in vivo (references in Ref. $3)$, the most likely explanation for the short-term transgene activity in normal human keratinocytes, either in culture or after transplantation on athymic animals, is the inefficient transduction of a stem cell population able to sustain the long-term turnover of the transduced cells.

\section{Identification and stable transduction of epidermal stem cells}

The clonogenic and growth potential of basal keratinocytes are two very different concepts. ${ }^{5}$ The former indicates the possibility of a basal cell to generate a colony, the latter deals with the self-renewal capacity, hence the 'stem-ness', of that cell.

Using clonal analysis, three types of keratinocytes with different capacities for multiplication have been identified and isolated in human epidermis, ie holoclones, meroclones and paraclones. ${ }^{5}$

Holoclones form large colonies $\left(1-2 \times 10^{5}\right.$ cells per each colony after 14 days of culture) with a smooth and regular perimeter formed by migrating involucrin-negative cells. When subcultivated, holoclones generate large and smooth daughter colonies. The holoclone has a tremendous growth capacity. Indeed, a single holoclone can generate more than $1 \times 10^{40}$ progeny (over 140 doublings), ie enough epithelia to cover several times an adult human body $\left(1.5-2 \mathrm{~m}^{2}\right)$, whose epidermis contains approximately $8 \times 10^{10}$ keratinocytes. ${ }^{3,5}$ Therefore, the basal keratinocyte generating a holoclone has the essential (and so far the only) characteristic in order to be considered as a stem cell, namely the extensive proliferative selfrenewal potential.

The paraclone, which is generated by a transient amplifying cell, has a limited growth potential, being committed to a maximum of 15 divisions (ie a maximum of $3 \times 10^{4}$ cells, covering an area of no more than $17 \mathrm{~mm}^{2}$ ). The colony founded by a paraclone is small and highly irregular, and its forming cells are large, squame-like and all involucrin-positive. When subcultivated, paraclones do not generate colonies, indicating that paraclones are formed by terminal cells. ${ }^{5}$

The meroclone is an intermediate type of cell and is considered to be a reservoir of transient amplifying cells. ${ }^{5}$

The transition from holoclone to meroclone to paraclone is a unidirectional process which occurs during natural ageing as well as during repeated keratinocyte subcultivation. Therefore, the aborted colonies generated by paraclones are actually the expression of keratinocyte senescence.

This said, a recent paper has shown that clonogenic keratinocytes with the characteristics of epidermal stem cells can be stably transduced with retroviral constructs expressing a bacterial $\beta$-galactosidase gene or a human IL-6 cDNA under control of a long terminal repeat. ${ }^{3}$ In particular, using clonal analysis, it has been shown that: (1) holoclones can be stably transduced by retroviral constructs; (2) the transgene expression lasts for the entire life-span of the culture (more than 150 cell generations); (3) keratinocyte stem cells integrate either one or multiple proviral copies in their genome; (4) the synthesis of the exogenous protein is proportional to the number of proviral integrations; and (5) when grafted on athymic animals, epidermal sheets generated from transduced clones secrete the exogenous protein in the blood circulation at levels strictly proportional to the rate of secretion in vitro and therefore to the number of proviral integrations. ${ }^{3}$

In these experiments, circulating IL-6 levels were maintained up to 7 days after grafting, the longest period during which the graft maintained a healthy stratified epithelium. This might be due to the unnatural position of the grafted epidermal sheet envisaged by the everted flap technique. ${ }^{3}$ Recently, loss of expression of a retrovirustransduced gene in human keratinocytes has been reported after grafting on to athymic animals. ${ }^{4}$ This could be due to either inactivation in vivo of the retroviral promoter or failure of stem cell transduction. Even though in vivo expression of retroviral promoters is still an open question, it is worth noting that retroviral vector inactivation was not observed in human haematopoietic cells after autologous transplantation on to patients or in transduced canine keratinocytes after autologous transplantation (references in Ref. 3), suggesting that when cells are grafted on to the same species/donor and/or in a normal position, the expression of the transgene can indeed be retained and that transcriptional inactivation of retroviral genomes occurs in mice through poorly identified mechanisms specific for certain cell types.

\section{Summary and perspectives}

Compared with other target cells of ex vivo gene therapy, human keratinocytes have many unique features: (1) keratinocytes can be easily cultivated and generate cohesive sheets of epithelium routinely used for grafting on to patients; (2) grafts are readily visible over time, allowing constant control and follow-up of the graft itself as well as the possibility of local modulation of the transgene expression using an appropriate promoter; (3) epidermal stem cells can be stably transduced allowing the longterm survival of the graft; and (4) transduced keratinocytes are able to secrete the exogenous gene product into the bloodstream.

These features reveal new perspectives in the long treatment of genetic skin disorders, as well as in the delivery of recombinant proteins in the circulation for gene therapy of systemic disorders.

However, a number of issues need to be addressed before considering gene therapy using keratinocytes: (1) tissue and 'stratum'-specific promoters should be used to drive properly the expression of specific genes (as laminin 5 or integrins); and (2) gene targeting technologies and/or recombinase-mediated integrative recombination strategies should be investigated in order to circumvent concerns linked with the random multiple integration of the transgenes into the keratinocyte genome occurring when retroviral vectors are used.

In terms of gene therapy for genetic skin diseases, the Herlitz type of EB may represent an unrealistic clinical challenge (given the early lethality and the involvement of several lining epithelia), while the non-Herlitz forms of junctional EB and the dystrophic type of EB are realistic clinical targets for gene therapy. Gene defects of most of the non-Herlitz type of junctional EB can be, in principle, corrected by retrovirus-mediated gene transfer. However, given the large number of molecules (such as 
type VII collagen) involved in the pathogenesis of the dystrophic type of EB, for gene correction of this particular form, conventional retroviral techniques for introduction of the wild-type cDNA need to be substituted by different and novel technologies such as chimeric RNA/DNA oligonucleotides for targeted gene correction or ribozyme-mediated repair of mutant mRNA by targeted trans-splicing.

Michele De Luca and Graziella Pellegrini Laboratory of Tissue Engineering IDI, Istituto Dermopatico dell'Immacolata Via dei Castelli Romani 83-85, 00040, Pomezia (Rome), Italy

\section{References}

1 Gallico GG et al. Permanent coverage of large burn wounds with autologous cultured human epithelium. New Engl J Med 1984; 311: 448-451.

2 Christiano AM, Uitto J. Molecular complexity of the cutaneous basement membrane zone. Exp Dermatol 1996; 5: 1-11.

3 Mathor MB et al. Clonal analysis of stably transduced human epidermal stem cells in culture. Proc Natl Acad Sci USA 1996; 93: 10371-10376.

4 Fenjves ES, Yao SN, Kurachi K, Taichman LB. Loss of expression of a retrovirus-transduced gene in human keratinocytes. J Invest Dermatol 1996; 106: 576-578.

5 Barrandon Y, Green H. Three clonal types of keratinocytes with different capacities for multiplication. Proc Natl Acad Sci USA 1987; 84: 2302-2306. 